\title{
A CLASS OF TRANSLATION PLANES AND A CONJECTURE OF D. R. HUGHES( $\left.{ }^{1}\right)$
}

\author{
BY
}

F. W. WILKE

1. Introduction. In [7] a class $(C)$ of right Veblen-Wedderburn systems (hereafter refered to as V-W systems) was defined which contains as a proper subclass the Andre V-W systems. (Some authors, in particular Andre [1], use the term quasi-field for a V-W system.) It was shown in [7] that members of a subclass of $(C)$, consisting of $\mathrm{V}-\mathrm{W}$ systems of order $3^{n}$ where $n$ is odd and not prime, are associated with translation planes which are not isomorphic to any Andre plane.

The main objective of this paper is to prove that the class $(C)$ contains a subclass $\left(C_{1}\right)$ of $\mathrm{V}-\mathrm{W}$ systems such that every collineation of the associated translation planes fixes both $X=(0)$ and $Y=(\infty)$. $\left(C_{1}\right)$ contains systems of order $p^{n}$ where $n$ is not prime if $p$ is odd and $n$ has a nonprime proper divisor if $p=2$. To accomplish this objective we prove some theorems concerning the collineations of planes associated with certain subclasses of $(C)$.

In [3] Foulser has recently defined a class of finite (left) V-W systems called $\lambda$-systems. It can be shown that these $\lambda$-systems and the finite systems of the subclass $\left(C^{*}\right)$ of $(C)$, defined in $\S 2$, determine the same class of translation planes. The class $(C)$ contains $\mathrm{V}$-W systems which are not $\lambda$-systems. In particular one may obtain a finite $(C)$ system by starting with the exceptional near-field of order 25 and using an additive mapping which is not an automorphism of the near-field. There are also infinite systems in $(C)$.

Let $F(+, \odot)$ be a right $\mathrm{V}-\mathrm{W}$ system (hereafter called simply a V-W system). Let $\pi$ be the projective plane associated with $F(+, \odot)$ as in [4, p. 353]. Then $\pi$ is a translation plane relative to the line $L_{\infty}=X Y$. Throughout this paper we will use the symbols $O, X, Y$ and $I$ to represent the points of $\pi$ with coordinates $(0,0),(0)$, $(\infty)$ and $(1,1)$ respectively. If $P$ and $Q$ are distinct points of $\pi, P Q$ will denote the line containing $P$ and $Q$. If $\alpha$ is a perspectivity of $\pi$ with center $P$ and axis $w, \alpha$ will be called a $P$ - $w$ perspectivity. The symbols $F_{\rho}, F_{\mu}$ and $F_{\lambda}$ will denote the right, middle and left nucleus respectively of the multiplicative loop $F^{\prime}(\odot)$ and $D=$ $\{a \in F \mid a \odot(x+y)=a \odot x+a \odot y$ for all $x, y \in F\}$. Let $K=F_{\lambda} \cap D$.

$\$ 2$ is devoted to showing that if a translation plane $\pi$ is associated with a V-W system in a certain subclass of $(C)$ then any collineation of $\pi$ either fixes $X$ and $Y$ or interchanges them. This result is used in $\$ 3$ to obtain the main result.

Received by the editors August 3, 1967 and, in revised form, May 21, 1968.

( ${ }^{1}$ ) This research was supported in part by a University of Missouri summer research grant. Cópyright (C) 1969, American Mathematical Society 
In [3] Foulser states that D. R. Hughes has conjectured the following: If $\pi$ is a finite Andre plane which has a collineation moving $X \rightarrow(m) \neq X, Y$ then $\pi$ is a Hall plane. In $\S 4$ we prove that this conjecture is correct.

2. The class $(C)$ of $\mathrm{V}-\mathrm{W}$ systems. The class $(C)$ of $\mathrm{V}-\mathrm{W}$ systems has been introduced in [7]. We will repeat the definition here for the convenience of the reader.

Let $F(+, \cdot)$ be a right near-field (that is a near-field with $(a+b) c=a c+b c$ for all $a, b, c \in F$ ) with additive identity 0 and multiplicative identity 1 and let $T$ be an automorphism of the additive group of $F$ with $1 T=1$. Let $F^{\prime}$ denote the set of nonzero elements of $F$ and let $\sigma: F^{\prime} \rightarrow Z$ be a mapping of $F^{\prime}$ into the integers such that for each $a, b \in F^{\prime}$ there is a unique integer $m$ such that $m=\sigma\left(\left(a T^{m}\right)^{-1} b\right)$. If $\sigma(1)=0$ and an operation $\odot$ is defined on $F$ by $x \odot y=x T^{\sigma(y)} y$ for $x, y \in F^{\prime}$ and $x \odot 0=0 \odot x=0$ for all $x \in F$, where we write $z w$ for $z \cdot w$, then it is not difficult to show that the system $F(+, \odot)$ is a right $\mathrm{V}-\mathrm{W}$ system, if $F(+)$ is finite dimensional over $K$.

Definition 1. The class of all V-W systems obtainable from nearfields in the manner described above will be called class $(C)$. The subclass of $(C)$ obtained by assuming that $F(+, \cdot)$ is a field and that $T$ is an automorphism of $F(+, \cdot)$ will be called class $\left(C^{*}\right)$. We will call a $\mathrm{V}-\mathrm{W}$ system proper if it is not a field.

It has been shown in [7] that each finite Andre system is in class $(C)$ and it is then clear that each finite Andre system is also in class $\left(C^{*}\right)$. [For a description of the finite Andre systems see ([1], [5], or [7]).]

Theorems 1 and 2 may be found in [7] and [3].

Theorem 1. Let $F(+, \cdot)$ be $a V-W$ system and let $\pi$ be the associated translation plane. $\pi$ has a $Y$-O Y perspectivity $\beta$ with $X \beta=(m)$ for $m \in F$ if and only if $a \cdot(m+b)$ $=a \cdot m+a \cdot b$ for all $a, b \in F$.

TheOREM 2. Let $F(+, \cdot)$ be a $V-W$ system and let $\pi$ be the associated translation plane. There is a one-to-one correspondence between

(a) the elements of $F_{\rho}$ and the $Y-O X$ perspectivities of $\pi$;

(b) the elements of $F_{\mu}$ and the $X-O Y$ perspectivities of $\pi$; and

(c) the elements of $K$ and the $O-X Y$ perspectivities of $\pi$.

THEOREM 3. Let $F(+, \odot)$ be a $V$-W system in $\left(C^{*}\right)$. If there is an element $m \in F$, $m \neq 0$, such that $a \odot(m+b)=a \odot m+a \odot b$ for all $a, b \in F$ then $F(+, \odot)$ is a field.

COROLlaRY. If $F(+, \odot)$ is a proper $V$-W system in $\left(C^{*}\right)$ and $\pi$ is the associated translation plane then $\pi$ has no nonidentity $Y-O Y$ perspectivity.

Proof. The proof given here is essentially the proof given by Andre to show that a proper Andre V-W system does not satisfy the left distributive law.

Let $F(+, \cdot)$ and $T$ be the field and automorphism respectively used in the construction of $F(+, \odot)$. Suppose $a \odot(m+b)=a \odot m+a \odot b$ for all $a, b \in F$ 
where $m \neq 0$. Then $a T^{\sigma(m+b)}=\left[a T^{\sigma(m)} m+a T^{\sigma(b)} b\right](m+b)^{-1}$ for all $a, b \neq-m \in F^{\prime}$ $=F-\{0\}$. Since $T$ is an automorphism we then have

$$
\begin{aligned}
\left(a^{2}\right) T^{\sigma(m+b)} & =\left[\left(a^{2}\right) T^{\sigma(m)} m+\left(a^{2}\right) T^{\sigma(b)} b\right](m+b)^{-1} \\
& =\left\{\left[a T^{\sigma(m)} m+a T^{\sigma(b)} b\right](m+b)^{-1}\right\}^{2}
\end{aligned}
$$

for all $a, b \neq-m \in F^{\prime}$. A straightforward calculation now shows that $a T^{\sigma(m)}=a T^{\sigma(b)}$ for all $a, b \neq-m \in F^{\prime}$ and hence $\sigma(m)=\sigma(b)$ for all $b \neq-m \in F^{\prime}$. If $b=-m$ then we also get $\sigma(m)=\sigma(b)$. Thus $\sigma(b)=\sigma(m)$ for all $b \in F^{\prime}$. Taking $b=1$ we see that $\sigma(m)=0$ and the theorem is proved.

The corollary is now an immediate consequence of Theorems 1 and 3.

THEOREM 4. Let $F(+, \odot)$ be a $V-W$ system in $\left(C^{*}\right)$ and let $\pi$ be the associated translation plane. If $\pi$ is recoordinatized by leaving the coordinates of OI unchanged and interchanging the roles of $X$ and $Y$ then the new $V$ - $W$ system so obtained is in $\left(C^{*}\right)$. Also, if $F(+, \odot)$ is proper then so is the new system.

Corollary. If $F(+, \odot)$ is a proper $V-W$ system in $\left(C^{*}\right)$ and $\pi$ is the associated translation plane then $\pi$ has no nonidentity $X-O X$ perspectivity.

Proof. It is not difficult to show that if $\oplus$ and $\otimes$ denote the addition and multiplication respectively in the new system then $x \oplus y=x+y$ for all $x, y \in F$ and $x \otimes y=x R^{-1}(y J)$ for all $x, y \neq 0 \in F$ where $y \odot y J=1$ and $z R(y J)=z \odot y J$. Thus $x \otimes y=x T^{-\sigma(y J)} y$ and $F(+, \otimes)$ is in $\left(C^{*}\right)$. Clearly, if $F(+, \odot)$ is proper then $F(+, \otimes)$ is proper.

The corollary follows immediately from the theorem and the corollary to Theorem 3.

Throughout the remainder of this section let $F(+, \odot)$ be a finite $\mathrm{V}-\mathrm{W}$ system in $\left(C^{*}\right)$ of order $\neq 2^{6}, 3^{2}$ and let $\pi$ be the associated translation plane. For each $x \in F_{\rho}$ let $\eta(x)$ be the $Y-O X$ perspectivity determined by the following mapping of the points of $\pi$ onto the points of $\pi$, (see [7, Theorem 7]); $(c, d) \eta(x)=(c, d \odot x)$, $(m) \eta(x)=(m \odot x), Y \eta(x)=Y$.

THEOREM 5. $F_{\rho}$ and $F_{\mu}$ are each nontrivial.

Proof. Let $u=\operatorname{LCM}\left\{p^{k}-1 \mid 0<k<n\right.$ and $\left.k \mid n\right\}$. Let $\rho$ be a generator of the multiplicative group of the field $G F\left(p^{n}\right)$. If $i \equiv j \bmod u$ then $\sigma\left(\rho^{i}\right)=\sigma\left(\rho^{j}\right)$ for otherwise there exists an integer $k$ such that $\rho^{k} \odot \rho^{i}=\rho^{k} \odot \rho^{j}$. Thus, $(1) \neq\left(\rho^{u}\right) \subset F_{\rho} \cap F_{\mu}$.

THEOREM 6. No collineation of $\pi$ fixes one of $X$ and $Y$ and moves the other.

Proof. (See [3, Lemma 6.1].) By the corollary to Theorem $3 \pi$ has no nonidentity $Y-O Y$ perspectivity. Thus, using [2, Theorem 3], since $\pi$ has nonidentity $X-O Y$ perspectivities, $\pi$ has no collineation fixing $Y$ and moving $X$. Using Theorem 4 we also see that no collineation of $\pi$ fixes $X$ and moves $Y$.

THEOREM 7. If $a, b \in F^{\prime}$ and $\sigma(a) \neq \sigma(b)$ then there is a collineation $\gamma$ of $\pi$ such that $(a) \gamma=(a)$ and $(b) \gamma \neq(b)$. 
COROLlaRY. If $\delta$ is a collineation of $\pi$ such that $Y \delta=(a) \neq(0)$ and $X \delta=(b) \neq(0)$ then $\sigma(a)=\sigma(b)$.

Proof. Let $\rho$ and $u$ be defined as in Theorem 5 and let $g=\rho^{u}$. Then, from the proof of Theorem 5, $g \in F_{\rho} \cap F_{\mu}$ and therefore $g T^{-\sigma(a)} \in F_{\mu}$. Let $\beta$ be the $X-O Y$ perspectivity defined by:

$$
\begin{aligned}
(c, d) \beta & =\left(c \odot g T^{-\sigma(a)}, d\right) \\
(m) \beta & =\left(g^{-1} T^{-\sigma(a)} \odot m\right) \\
Y \beta & =Y .
\end{aligned}
$$

Let $\gamma=\eta(g) \beta$. Then

$$
(a) \gamma=(a \odot g) \beta=\left(g^{-1} T^{-\sigma(a)} \odot(a \odot g)\right)=(a)
$$

and

$$
\begin{aligned}
(b) \gamma & =(b \odot g) \beta=\left(g^{-1} T^{-\sigma(a)} \odot(b \odot g)\right) \\
& =\left(\left(g^{-1} T^{-\sigma(a)} \odot b\right) \odot g\right) \\
& =\left(\left(g^{-1} T^{[\sigma(b)-\sigma(a)]} g\right) b\right)
\end{aligned}
$$

since $\sigma(g)=0$. Thus, $(b) \gamma=(b)$ if and only if $T^{[\sigma(b)-\sigma(a)]}$ fixes $g^{-1}$ and hence fixes $g$, which is impossible by the choice of $u$. (See [3].)

To prove the corollary suppose $Y \delta=(a) \neq(0)$ and $X \delta=(b) \neq(0)$ and $\sigma(a) \neq \sigma(b)$. Then $\delta \gamma \delta^{-1}$ fixes $Y$ and moves $X$ contrary to Theorem 6 .

THEOREM 8. If $\gamma$ is a collineation of $\pi$ such that $Y \gamma=(r), X \gamma=(s) \neq X$ then there is a collineation $\delta$ of $\pi$ such that $Y \delta=\left(r_{1}\right), X \delta=\left(s_{1}\right) \neq X$ and $r_{1}+s_{1} \neq 0$.

Proof. Let $\delta=\gamma^{-1} \eta(g) \gamma$ where $g$ is as in Theorem 7. Then $\delta$ is an $(r)-O(s)$ perspectivity of $\pi$. If $Y \delta=\left(r_{1}\right)$ and $X \delta=\left(s_{1}\right)$ then a straightforward calculation shows that $Y \delta^{-1}=\left(-r_{1}+r+s\right)$. If $r+s=0=r_{1}+s_{1}$ then $Y \delta^{-2}=\left(-r_{1}\right) \delta^{-1}=\left(s_{1}\right) \delta^{-1}$ $=X$ so that $\delta$ has order 4 . But $O(\delta)=\left(p^{n}-1\right) / u=4$ implies $p=3$ and $n=2$. Thus, either $r+s \neq 0$ or $r_{1}+s_{1} \neq 0$.

THEOREM 9. Let $\gamma$ be a collineation of $\pi$ such that $Y \gamma=(r), X \gamma=(s) \neq X$ and $r+s \neq 0$. If $\delta$ is an $(r)-O(s)$ perspectivity of $\pi$ with $Y \delta=(a) \neq X$ and $X \delta=(b)$ then $\sigma(r)=\sigma(s)=\sigma(a)=\sigma(b)$ and $a b+r s=b(r+s)$.

Proof. From the proof of Theorem 8 we have

$$
Y \delta^{-1}=(-a+r+s)
$$

so that

$$
(x, x \odot(-a+r+s)) \delta=(0, y)
$$

for some $y \in F$. Thus,

$$
x+[x \odot(-a+r+s)-x \odot s] Q^{-1}=0
$$

where $z Q=z \odot a-z \odot r$ for all $z \in F$. From this we get

$$
x \odot(-a+r+s)=-(x \odot a)+x \odot r+x \odot s
$$


for all $x \in F$. By the corollary to Theorem 7 we have $\sigma(r)=\sigma(s)$ and $\sigma(a)=\sigma(b)$. Therefore

$$
\left[x T^{\sigma(-a+r+s)}-x T^{\sigma(r)}\right](r+s)=\left[x T^{\sigma(-a+r+s)}-x T^{\sigma(a)}\right] a
$$

for all $x \in F$.

Suppose $\sigma(a) \neq \sigma(r)$. Then by Theorem 7 there is a collineation $\alpha$ of $\pi$ such that $(r) \alpha=(r)$ and $(a) \alpha \neq(a)$. From the proof of Theorem 7 we have, with $g=\rho^{u}$ as before,

$$
(m) \alpha=\left(m\left[g^{-1} T^{[\sigma(m)-\sigma(r)]} g\right]\right)
$$

for $m \in F$ and $Y \alpha=Y$. Let $\beta=\alpha^{-1} \delta \alpha$. Then $\beta$ is an $(r)-O(s)$ perspectivity of $\pi$ with $Y \beta=(a) \alpha=\left(a g_{1}\right)$ and

$$
Y \beta^{-1}=(-a+r+s) \alpha=\left([-a+r+s] g_{2}\right)=(z)
$$

where $g_{1}=g^{-1} T^{[\sigma(a)-\sigma(r)]} g$ and $g_{2}=g^{-1} T^{[\sigma(-a+r+s)-\sigma(r)]} g$.

Then from (1)

$$
\left[x T^{\sigma(z)}-x T^{\sigma(r)}\right](r+s)=\left[x T^{\sigma(z)}-x T^{\sigma\left(a g_{1}\right)}\right]\left(a g_{1}\right) .
$$

Now $\sigma(z)=\sigma(-a+r+s)$ and $\sigma\left(a g_{1}\right)=\sigma(a)$ so that the left sides of (1) and (2) are identical.

Therefore

$$
\left[x T^{\sigma(z)}-x T^{\sigma(a)}\right]\left(g_{1}-1\right) a=0
$$

for all $x \in F$.

Since $a \neq 0$ we have either $g_{1}=1$ or $x T^{\sigma(z)}=x T^{\sigma(a)}$ for all $x \in F$.

The choice of $u$ insures that $g_{1} \neq 1$ so we must have $\sigma(z)=\sigma(a)$. But then since $r+s \neq 0$ we also have $\sigma(r)=\sigma(z)=\sigma(a)$.

A straightforward calculation shows that

$$
\left(-(a-r) R^{-1}(-a+r+s), 0\right) \delta=\left(1,\left[(a-r) R^{-1}(-a+r+s) \odot s\right] Q^{-1} \odot r\right)
$$

where $x R(y)=x \odot y$. Since $X \delta=(b)$ we have

$$
b=\left[(a-r) R^{-1}(-a+r+s) \odot s\right] Q^{-1} \odot r .
$$

From this, noting that $\sigma(a)=\sigma(r)=\sigma(-a+r+s)$ implies that

$$
x Q^{-1}=\left[x(a-r)^{-1}\right] T^{-\sigma(r)},
$$

it follows easily that $a b+r s=b(r+s)$.

THEOREM 10. If $F_{\rho}$ has order $t$ and $t[t / 2]>p^{n}-1$ then any collineation of $\pi$ either fixes $X$ and $Y$ or interchanges them.

COROLlaRY 1. If there exists $k<n / 2$ such that $k \mid n$ and $\left(p^{n}-1\right) /\left(p^{k}-1\right) \leqq t$ then any collineation of $\pi$ either fixes $X$ and $Y$ or interchanges them. 
Proof. Let $g_{1}, g_{2}, \ldots, g_{t}=1$ be the elements of $F_{\rho}$ where $o\left(g_{i}\right)>2, i \leqq h<t$ and let $\eta\left(g_{i}\right)$ be the $Y-O X$ perspectivity associated with $g_{i}$. Suppose $\gamma$ is a collineation of $\pi$ such that $Y \gamma=(r)$ and $X \gamma=(s)$. By Theorem 8 we may assume $r+s \neq 0$. Let $\delta_{i}=\gamma^{-1} \eta\left(g_{i}\right) \gamma$ and $\beta_{i}\left(g_{j}\right)=\eta\left(g_{j}\right)^{-1} \delta_{i} \eta\left(g_{j}\right)$. If $Y \delta_{i}=\left(a_{i}\right)$ and $X \delta_{i}=\left(b_{i}\right)$ then $Y \beta_{i}\left(g_{j}\right)$ $=\left(a_{i} \odot g_{j}\right)$ and $X \beta_{i}\left(g_{j}\right)=\left(b_{i} \odot g_{j}\right)$.

Suppose $a_{i} \odot g_{j}=a_{m} \odot g_{f}$ for some $i \neq m, j, f$. Then $a_{i}=a_{m} \odot g_{w}$ for some $1 \leqq w \leqq t$. $\sigma\left(a_{i}\right)=\sigma\left(a_{m}\right)$ and $g_{w} \in F_{\rho}$ imply $\sigma\left(g_{w}\right)=0$, so that $a_{i}=a_{m} g_{w}$ and $b_{i}=b_{m} g_{w}$.

By Theorem 9 we have $a_{i} b_{\imath}+r s=b_{i}(r+s)$ so that

$$
a_{m} b_{m} g_{w}^{2}+r s=b_{m} g_{w}(r+s)=g_{w}\left(a_{m} b_{m}+r s\right) \text {. }
$$

Therefore $g_{w}=1$ or $g_{w}=r s a_{m}^{-1} b_{m}^{-1}$. Since $i \neq m, g_{w} \neq 1$ so that $a_{i}=a_{m} g_{w}=r s b_{m}^{-1}$ $=-a_{m}+r+s$. Thus, $Y \delta_{i}=Y \delta_{m}^{-1}$ or $\delta_{i}=\delta_{m}^{-1}$. We may assume that the $\delta_{j}$ 's are listed so that $\delta_{j}^{-1}=\delta_{h / 2+j}$ for $j \leqq h / 2$. Let $B=\{1,2, \ldots, h / 2, h+1, \ldots, t-1\}$. Then if $i$, $m \in B, i \neq m$, then $\delta_{i} \neq \delta_{m}^{-1}$.

Let $A=\left\{a_{i} \odot g_{j} \mid i \in B, 1 \leqq j \leqq t\right\}$. Then, if $|C|$ denotes the number of elements in a set $C,|A|=|B| t \geqq[t / 2] t$. But the elements of $A$ are coordinates of points on $X Y$ each of which is different from each of $X, Y$. Therefore, if the collineation $\gamma$ exists then the line $X Y$ contains more than $p^{n}+1$ points which is impossible.

The corollary is an immediate consequence of the theorem.

Definition 2. A V-W system in $\left(C^{*}\right)$ which satisfies the hypothesis of Theorem 10 will be called a special $\left(C^{*}\right)$ system.

Corollary 2. Let $F(+, \bigcirc)$ be an Andre system and let $S$ be the automorphism used in the construction of $F(+, \odot)$. If order $(S)>2$ then $F(+, \odot)$ is a special $\left(C^{*}\right)$ system.

Proof. Let $H=\{x \in F \mid \nu(x)=1\}$. Then $H$ is a cyclic subgroup of $F_{o}$ of order $\left(p^{n}-1\right) /\left(p^{n / s}-1\right)$ where order $(S)=s$. Since $s>2$ we have $n / s<n / 2$ and the result follows from Corollary 1.

3. Some new V-W systems and their associated translation planes. Let $p$ be a prime, $F(+, \cdot)=G F\left(p^{n}\right)$ and let $k(0)=n, k(1), \ldots, k(j)$ be positive integers such that $k(j)<k(j-1)<\cdots<k(0)$ and $k(i)$ divides $k(i-1)$ for $1 \leqq i \leqq j$. If $p=2$ then we add the restriction that $k(j)>1$. Let $\rho$ be a generator of the multiplicative group of $F(+, \cdot)$ and for each $0 \leqq i \leqq j$ define

$$
G_{i}=\left(\rho^{\left[p^{k(i)}-1\right]}\right) .
$$

Then (1) $=G_{0} \subset G_{1} \subset \ldots \subset G_{j} \subset F^{\prime}(\cdot)$ is a strictly increasing sequence of subgroups of $F^{\prime}(\cdot)$. Define $\sigma$ by

$$
\begin{aligned}
\sigma(x) & =k(0) \quad \text { if } x \in G_{1} \\
& =k(i) \quad \text { if } x \in G_{i+1}-G_{i}, 1 \leqq i<j \\
& =k(j) \quad \text { if } x \notin G_{j}
\end{aligned}
$$


and define an operation $\odot$ on $F$ by

$$
\begin{aligned}
x \odot y & =x T^{\sigma(y)} \cdot y & & \text { if } y \neq 0 \\
& =0 & & \text { if } y=0
\end{aligned}
$$

where $x T=x^{p}$ for all $x \in F$. It is easily seen that $F(+, \odot)$ is a V-W system in $\left(C^{*}\right)$.

Definition 3. The subclass of $\left(C^{*}\right)$ consisting of all V-W systems obtained in the above manner with $j \geqq 2$ will be denoted by $\left(C_{1}\right)$. If $p=3$ and $n$ is even then we also add the condition that $k(j)>1$.

THEOREM 11. If $F(+, \odot)$ is a $\left(C_{1}\right)$ system then $F_{\rho}=F_{\mu}=G_{1}$.

COROLlaRY. If $k(1)<n / 2$ then $a\left(C_{1}\right)$ system is a special $\left(C^{*}\right)$ system.

Proof. A simple calculation shows that $a \in F_{\rho}$ if and only if

for all $y \in F^{\prime}$.

$$
\sigma(y \odot a) \equiv[\sigma(y)+\sigma(a)] \bmod n
$$

If $a \in G_{1}$ then $\sigma(y \odot a)=\sigma(y a)=\sigma(y)=\sigma(y)+\sigma(a)$ for all $y \in F^{\prime}$ so that $G_{1} \subset F_{\rho}$.

Suppose $a \notin G_{1}$. Then $a \in G_{i+1}-G_{i}$ for some $1 \leqq i<j$ or $a \notin G_{j}$. If $a \in G_{i+1}-G_{i}$ let $y \in F^{\prime}-G_{j}$. Then $y \odot a \notin G_{j}$ and $\sigma(y \odot a)=k(j)$ while $\sigma(y)+\sigma(a)=k(j)+k(i)$. Thus $a \in F_{\rho}$ would imply that $k(i)=n$ which implies $a \in G_{1}$. If $a \notin G_{j}$ let $y \in G_{2}-G_{1}$. Again $y \odot a \notin G_{j}$ so that $\sigma(y \odot a)=k(j)$ while $\sigma(y)+\sigma(a)=k(j)+k(i)$. Therefore $F_{\rho} \subset G_{1}$ and we have $F_{\rho}=G_{1}$.

Similarly $F_{\mu}=G_{1}$.

The proof of the corollary is immediate since $\rho^{p^{k(1)}-1} \in G_{1}$.

THEOREM 12. If $F(+, \odot)$ is a $\left(C_{1}\right)$ system then

(1) $._{\lambda}=\left\{x \in F^{\prime} \mid x T^{k(j)}=x\right\}$,

(2) $F_{\lambda} \subset D$.

Proof. Let $L=\left\{x \in F^{\prime} \mid x T^{k(j)}=x\right\} \cdot a \in F_{\lambda}$ if and only if

$$
a T^{\sigma(x \odot y)}=a T^{[\sigma(x)+\sigma(y)]}
$$

for all $x, y \in F^{\prime} . \rho \notin G_{j}$ and $\rho \odot \rho \notin G_{j}$ so that $\sigma(\rho \odot \rho)=\sigma(\rho)=k(j)$. Thus, $a \in F_{\lambda}$ implies

$$
a T^{k(j)}=a T^{[k(j)+k(j)]}
$$

so that $a=a T^{k(j)}$. Since $k(j)$ divides $k(i)$ for $i<j$ we see that if $a \in L$ then $a \in F_{\lambda}$. Thus, $L=F$.

Finally if $a \in F_{\lambda}$ then $a \odot(x+y)=a(x+y)=a x+a y=a \odot x+a \odot y$ so that $F_{\lambda} \subset D$.

Two V-W systems $F_{1}(+, \odot)$ and $F_{2}(\oplus, \otimes)$ are said to be isotopic if there exists three one-to-one mappings $\alpha, \beta$ and $\gamma$ of $F_{1}$ onto $F_{2}$ such that $O \gamma=O$ and

$$
(x \odot y+z) \gamma=x \alpha \otimes y \beta \oplus z \gamma
$$

for all $x, y, z \in F_{1}$. It is easily seen that $(x \odot y) \gamma=(x \odot a) \gamma \otimes(b \odot y) \gamma$ where 
$a=1 \beta^{-1}$ and $b=1 \alpha^{-1}$ and 1 is the identity for $\otimes$. Foulser, [3, Proposition 5.3], has shown that for $\left(C^{*}\right)$ systems $z \gamma=\left[z(b \odot a)^{-1}\right] \eta$ for every $z \in F_{1}$ where $\eta$ is an automorphism of $F_{1}$. (Note that here we may assume $F_{1}=F_{2}$.) It is known (see [6]) that isotopic V-W systems are associated with isomorphic translation planes while the converse is not true. It is also known that if $F_{1}$ and $F_{2}$ are $\mathrm{V}-\mathrm{W}$ systems and $\pi_{1}$ and $\pi_{2}$ are the associated translation planes and if $\delta$ is an isomorphism from $\pi_{1}$ to $\pi_{2}$ with $O_{1} \delta=O_{2}, X_{1} \delta=X_{2}$ and $Y_{1} \delta=Y_{2}$ then $F_{1}$ is isotopic to $F_{2}$. This result will be refered to as Knuth's Theorem on isotopy.

THEOREM 13. If $F_{1}(+, \bigcirc)$ is a $\left(C_{1}\right)$ system and $F_{1}^{*}(\oplus, \otimes)$ is the $\left(C^{*}\right)$ system associated with $F_{1}(+, \bigcirc)$ as in Theorem 4 then $F_{1}$ and $F_{1}^{*}$ are not isotopic.

COROLlARY. If $F_{1}$ is a $\left(C_{1}\right)$ system and $\pi$ is the associated translation plane then no collineation moves $X$ or $Y$.

Proof. It is easily seen that in a $\left(C_{1}\right)$ system $\sigma(y J)=\sigma\left(y^{-1}\right)=\sigma(y)$ so that from the proof of Theorem 4 we have

$$
x \otimes y=x T^{-\sigma(y)} y .
$$

If $F_{1}$ and $F_{1}^{*}$ are isotopic then from the remarks above and a straightforward calculation there exist elements $a, b \in F_{1}$ such that

$$
-\sigma(x) \equiv \sigma(a)+\sigma\left(x a^{-1}\right) \bmod n
$$

for all $x \in F_{1}, x \neq 0$. Letting $x=a$ we have $-\sigma(a) \equiv \sigma(a)$ so that $\sigma(a)=n$ or $\sigma(a)=n / 2$. If $\sigma(a)=n$ then $-\sigma(x) \equiv \sigma(x)$ for all $x$. This is impossible since there exists an $x$ in $F_{1}$ such that $\sigma(x)<n / 2$. If $\sigma(a)=n / 2$ then $k(1)=n / 2$ and $a \in G_{2}-G_{1}$. Choose $x$ not in $G_{2}$. Then $\sigma\left(x a^{-1}\right)=\sigma(x)<n / 2$ so that $2 \sigma(x)+n / 2<n$. Therefore $F_{1}$ and $F_{1}^{*}$ are not isotopic.

The corollary is an immediate consequence of this theorem together with Theorem 10.

THEOREM 14. Let $F_{1}(+, \odot)$ be a $\left(C_{1}\right)$ system and let $F_{2}(\oplus, \otimes)$ be a $V-W$ system with $\pi_{1}$ and $\pi_{2}$ the associated translation planes. If either $F_{2 \rho}$ or $F_{2 \mu}$ is nontrivial then $\pi_{1}$ is isomorphic to $\pi_{2}$ if and only if $F_{2}$ is isotopic to $F_{1}$ or to $F_{1}^{*}$.

COROllary. $\pi_{1}$ is not isomorphic to any Andre plane.

Proof. The sufficiency is clear from Knuth's Theorem on isotopy.

If $\eta$ is an isomorphism from $\pi_{2}$ to $\pi_{1}$ then since at least one of $F_{2 \rho}$ or $F_{2 \mu}$ is nontrivial $\pi_{1}$ has a collineation which moves $X$ and $Y$ or $F_{2}$ is isotopic to one of $F_{1}$ or $F_{1}^{*}$. By the corollary to Theorem 13 the first condition cannot hold and the theorem is proved.

To prove the corollary it is sufficient to prove that $F_{1}$ is not isotopic to any Andre system. Note that if $F_{1}^{*}$ is isotopic to the Andre system $F_{2}$ then $F_{1}$ is isotopic to the Andre system $F_{2}^{*}$. 
If the $\left(C_{1}\right)$ system $F_{1}(+, \odot)$ is isotopic to the Andre system $F_{2}(\oplus, \otimes)$ then their multiplicative loops are isotopic so that $F_{1 \lambda}, F_{1 \mu}$ and $F_{1 \rho}$ are isomorphic to $F_{2 \lambda}$, $F_{2 \mu}$ and $F_{2 \rho}$ respectively. From Theorem 12 we have order $\left(F_{1 \lambda}\right)=p^{k(j)}-1$ where $k(j)<k(1)$ and $k(j)$ divides $k(1)$. Let $S$ be the automorphism of $G F\left(p^{n}\right)$ used to construct $F_{2}$ so that $x \otimes y=x S^{\mu v(y)} y$. If $a S=a$ then $a \in F_{2 \lambda}$. Therefore the fixed field of $S$ has order $\leqq p^{k(j)}$ and $S$ has order $\geqq n / k(j)$. If $g \in F_{2}$ and $\nu(g)=1$ then $g \in F_{2 \rho}$. Let $H=\left\{g \in F_{2} \mid \nu(g)=1\right\}$. Then order $(H)=\left(p^{n}-1\right) /\left(p^{n / t}-1\right)$ where order $(S)=t$. Now, $n / t \leqq k(j)<k(1)$ so that $\operatorname{order}(H)>\left(p^{n}-1\right) /\left(p^{k(1)}-1\right)=\operatorname{order}\left(G_{1}\right)$ $=\operatorname{order} F_{1 \rho}$. Since $H \subset F_{2 \rho}$ we have a contradiction and hence $F_{1}$ is not isotopic to $F_{2}$.

4. A conjecture of D. R. Hughes. We know from Corollary 2 to Theorem 10 that the only Andre systems which can have collineations moving $X$ and $Y$ without interchanging them are of order $p^{2 n}$ and the automorphism used in their construction must be of order 2. The next theorem determines all such Andre systems.

TheOREM 15. Let $F(+, \odot)$ be an Andre $V$-W system of order $p^{2 n}$, let $\pi$ be the associated translation plane and let $S$ be the automorphism of the field $G F\left(p^{2 n}\right)$ of order 2. If $\pi$ has a collineation $\gamma$ such that $X \gamma \neq X, Y$ then $\mu: k_{1} \rightarrow 0,(1), k \rightarrow 1,(0)$ for $k \neq k_{1}$, where $k, k_{1}$ are nonzero elements in the fixed field of $S$. That is, $\mu$ takes on one of the values 0,1 at exactly one $0 \neq k \in K$ and the other value at the rest of the nonzero elements of $K$.

Proof. Let $Y \gamma=(r) \neq X, Y$ and $X \gamma=(s) \neq X, Y$, and let $\delta$ be a $(r)-O(s)$ perspectivity of $\pi$, with $Y \delta=(a), X \delta=(b) \neq X, Y$. By Theorem $9 \mu \nu(r)=\mu \nu(s)=\mu \nu(a)$ $=\mu \nu(b)$.

For each $x \in F^{\prime}$ define the mapping $\beta_{x}$ as follows:

$(c, d) \beta_{x}=(x c, x d)$, where $x c$ is multiplication in $G F\left(p^{2 n}\right)$;

(m) $\beta_{x}=\left(\left[\left(x^{-1}\right) S^{\mu v(m)} x\right] m\right)$;

$Y \beta_{x}=Y$.

It is easily seen that $\beta_{x}$ is a collineation of $\pi$. Choose $x \in F^{\prime}$ such that $x^{-1} S x=g \neq 1$ and note that $g \in F_{\rho}$. For this $x$ denote $\beta_{x}$ simply by $\beta$.

Case 1. Suppose $\mu \nu(r)=1$. Then $(r) \beta=(r g)$ and $(s) \beta=(s g)$ so that $\beta^{-1} \delta \beta$ is a $(r g)-O(s g)$ perspectivity of $\pi$.

If $\eta(g)$ is the mapping $(x, y) \rightarrow(x, y g),(m) \rightarrow(m g) Y \rightarrow Y$ then $\eta(g)$ is a $Y$-OX perspectivity of $\pi$. Now,

$$
Y \beta^{-1} \delta \beta=(a) \beta=(a g)=Y \eta(g)^{-1} \delta \eta(g)
$$

so that $\beta^{-1} \delta \beta=\eta(g)^{-1} \delta \eta(g)$ since both are $(r g)-O(s g)$ perspectivities.

Suppose $m \in F$ such that $\mu \nu(m)=0$. Compute $(1, m) \beta^{-1} \delta \beta=(1, m) \eta(g)^{-1} \delta \eta(g)$. Then a straightforward, but somewhat lengthy, calculation shows that

$$
m S m=\nu(m)=\left(r s b^{-1}\right) S\left(r s a^{-1}\right) .
$$

Since $\mu \nu(1)=0$ we see that $\mu(k)=0$ if and only if $k=1$. 
Case 2. Suppose $\mu \nu(r)=0$. Then $(r) \beta=(r)$ and $(s) \beta=(s)$ so that $\beta^{-1} \delta \beta$ is an $(r)-O(s)$ perspectivity. Since $Y \beta^{-1} \delta \beta=(a) \beta=(a)$ we see that $\beta^{-1} \delta \beta=\delta$. If $\mu \nu(m)=1$ then essentially the same calculation as above yields again

$$
m S m=v(m)=\left(r s b^{-1}\right) S\left(r s a^{-1}\right)=k_{1} \neq 1 .
$$

Thus, $\mu(k)=1$ if and only if $k=k_{1}$.

THEOREM 16. Let $F(+, \cdot)$ be $G F\left(p^{2 n}\right)$, S the automorphism of $F$ of order 2 and let mappings $\mu_{1}, \mu_{2}$ be defined as follows:

$\mu_{1}: 1 \rightarrow 0,0 \rightarrow 0, k \rightarrow 1, k \neq 0,1 \in K$.

$\mu_{2}: k_{1} \rightarrow 1, k \rightarrow 0$ for $k_{1} \neq k \in K$ where $k_{1} \neq 1,0$.

If $x \odot y=x S^{\mu_{1} v(y)} y$ and $x \otimes y=x S^{\mu_{2} v(y)} y$ then $F(+, \odot)$ is isotopic to $F(+, \otimes)$.

Proof. $F(+, \odot)$ is isotopic to $F(+, \otimes)$ if and only if there exists an additive mapping $T$ from $F$ onto $F$ and elements $a, b \in F^{\prime}$ such that

$$
(x \odot y) T=(x \odot a) T \otimes(b \odot y) T .
$$

Choose $a$ such that $\nu(a)=k_{1}^{-1}$ and let $x T=x a^{-1}$. It is easily seen that

$$
(x \odot y) a^{-1}=(x \odot a) a^{-1} \otimes y a^{-1}
$$

and the theorem is proved.

$F(+, \odot)$ is the Andre system used by Albert and Hughes (see [3] for a proof) to prove that each Hall plane is an Andre plane. Since we have seen that the only Andre planes which can have a collineation moving $X \rightarrow(m) \neq X, Y$ are the ones described in Theorem 16 we see that Hughes' conjecture is correct.

\section{REFERENCES}

1. J. Andre, Über nicht-Desarguessche Ebenen mit transitiver translationsgruppe, Math. Z. 60 (1954), 156-186.

2. - Uber Perspektivitäten in endlichen projektiven Ebenen, Arch. Math. 6 (1954), 29-32.

3. D. Foulser, A generalization of Andre systems, Math. Z. 100 (1967), 380-395.

4. Marshall Hall, Jr., The theory of groups, Macmillan, New York, 1959.

5. D. R. Hughes, Review of some results in collineation groups, Proc. Sympos. Pure Math., vol. 1, Amer. Math. Soc., Providence, R. I., 1959.

6. D. E. Knuth, Finite semifields and projective planes, J. Algebra 2 (1965), 182-217.

7. F. W. Wilke and J. L. Zemmer, Some new finite translation planes, Trans. Amer. Math. Soc. 133 (1969), 378-397.

UNIVERSITY OF MISSOURI,

ST. LouIs, MissourI 